\title{
Interference resolution in major depression
}

\author{
JUTTA JOORMANN \\ University of Miami, Coral Gables, Florida \\ Derek Evan Nee, Marc G. Berman, and John Jonides \\ University of Michigan, Ann Arbor, Michigan \\ AND \\ IAN H. GothIB \\ Stanford University, Palo Alto, California
}

\begin{abstract}
In two experiments, we investigated individual differences in the ability to resolve interference in participants diagnosed with major depressive disorder (MDD). Participants were administered the "Ignore/Suppress" task, a short-term memory task composed of two steps. In Step 1 ("ignore"), participants were instructed to memorize a set of stimuli while ignoring simultaneously presented irrelevant material. In Step 2 ("suppress"), participants were instructed to forget a subset of the previously memorized material. The ability to resolve interference was indexed by response latencies on two recognition tasks in which participants decided whether a probe was a member of the target set. In Step 1, we compared response latencies to probes from the to-be-ignored list with response latencies to nonrecently presented items. In Step 2, we compared response latencies to probes from the to-be-suppressed list with response latencies to nonrecently presented items. The results indicate that, compared with control participants, depressed participants exhibited increased interference in the "suppress" but not in the "ignore" step of the task, when the stimuli were negative words. No group differences were obtained when we presented letters instead of emotional words. These findings indicate that depression is associated with difficulty in removing irrelevant negative material from short-term memory.
\end{abstract}

Recurrent, ruminative thoughts often experienced as unintentional and uncontrollable are among the most troubling aspects of depressive disorders. These thoughts commonly feature negative statements and pessimistic ideas about the self, the world, and the future; consequently, they likely play an important role in maintaining negative affect and, ultimately, in causing depressive episodes. Ruminative thoughts are not only a debilitating symptom of depression, they have also been associated both with vulnerability to the onset of depression and with the recurrence of depressive episodes (Nolen-Hoeksema, 2000; Nolen-Hoeksema, Wisco, \& Lyubomirsky, 2008). Despite the fact that over a decade of research has established the importance of rumination in depression, little is known about the cognitive processes that underlie the occurrence of ruminative thinking. In the present article, we investigate whether depression is associated with an inability to control the contents of short-term memory, and whether this impairment is related to rumination.

Short-term memory is a limited-capacity system that provides temporary access to a select set of representations in the service of current cognitive processes (Cowan, 1999; Miyake \& Shah, 1999). ${ }^{1}$ Thus, short-term memory reflects the focus of attention and the temporary activation of repre- sentations that are the contents of awareness (Jonides et al., 2008). Given the capacity limitation of this system, it is important that the contents of short-term memory be updated efficiently, a task controlled by executive processes (e.g., Friedman \& Miyake, 2004; Hasher, Zacks, \& May, 1999). Executive processes must selectively gate access to short-term memory, shielding it from intrusion from irrelevant material, as well as discard information that is no longer relevant. In this context, individual differences in the experience and resolution of interference are likely to affect cognitive and emotional functioning. The occurrence of intrusive thoughts might be one consequence of poor interference resolution. Indeed, increased interference from irrelevant representations has been proposed as a source of low short-term memory capacity (Engle, Kane, \& Tuholski, 1999) and has been found in various populations, including older adults (Hasher, Stoltzfus, Zacks, \& Rypma, 1991), children with attention deficit disorder (Bjorklund \& Harnishfeger, 1990), patients with obsessive-compulsive disorder (Enright \& Beech, 1990), and patients with schizophrenia (Frith, 1979). Therefore, it is noteworthy that several researchers have suggested that rumination and depression are associated with deficits in executive functioning (Hertel, 1997; Joormann, 2005; Linville, 1996). 
Deficits in controlling the contents of short-term memory may also affect depressed people's ability to regulate negative affect. The experience of negative mood is generally associated with, or in part consists of, the activation of mood-congruent representations in short-term memory (Isen, 1984; Siemer, 2005). Changes in cognition due to negative mood, however, are usually transient, and moodcongruent cognitions are often replaced quite quickly by thoughts and memories that serve to regulate and repair the mood state (Erber \& Erber, 1994; Parrott \& Sabini, 1990; Rusting \& DeHart, 2000). The ability to control the contents of short-term memory might therefore play an important role in recovery from negative mood. Thus, an inability to appropriately expel mood-congruent negative items from short-term memory as they become irrelevant would lead to difficulties attending to and processing new information, and might also result in rumination increasing the likelihood of a depressive episode.

Although the link between executive control and depression may help us understand the underlying mechanisms of rumination, it is important to note that the resolution of interference from irrelevant material is unlikely to be controlled by a single mechanism, and may differ according to the stage of processing of information at which interference occurs. Indeed, most contemporary theories postulate that executive control is not a unitary construct but, instead, involves several components such as the ability to focus attention, shift attention between stimuli, manage multiple tasks, and inhibit irrelevant processes or responses (e.g., Jonides \& Nee, 2005; Nee \& Jonides, 2008). In addition, these components of executive control operate at different stages of the processing of information (Hasher \& Zacks, 1988; Hasher et al., 1999). Hasher and Zacks, for example, differentiate interference resolution processes that keep unwanted information from entering short-term memory both from processes that rid shortterm memory of unwanted information and from processes that coordinate between prepotent responses and less prepotent responses. To investigate whether depression is associated with difficulties at all stages of information processing or, alternatively, to pinpoint the stage at which depressive deficits are most likely to occur, promises to help us gain a better understanding of cognitive deficits in depression and of the link between individual differences in the control over the contents of short-term memory and the occurrence of intrusive thoughts.

In addition to the question of whether depression affects all aspects of interference, there is the question of whether depression is associated with a general deficit in executive control or whether the deficit is specific to the processing of emotional material. Indeed, empirical evidence for a general deficit in short-term memory functioning in depression is inconsistent. For example, Channon, Baker, and Robertson (1993) found very few differences between depressed and nondepressed participants on a variety of short-term memory tasks (i.e., only on the backward digit span; see also Barch, Sheline, Csernansky, \& Snyder, 2003; Fossati, Amar, Raoux, Ergis, \& Allilaire, 1999; Purcell, Maruff, Kyrios, \& Pantelis, 1997). More recently, Rose and Ebmeier (2006) reported that depressed patients were slower and less accurate on an $n$-back task, and that task difficulty did not influence this effect. Harvey et al. (2004) did not find differences between depressed and nondepressed participants on any of a number of other tasks assessing short-term memory functioning, including digit span. In line with these findings, Egeland et al. (2003) concluded that reduced performance on short-term memory tasks in depression is due to a nonspecific speed reduction and to a loss of vigilance, factors consistent with a lack of effort but not with a specific deficit in executive functioning.

Similarly, Grant, Thase, and Sweeney (2001) administered a battery of cognitive tasks to 123 depressed outpatients and noted the surprising absence of cognitive deficits in their sample. The only indications of deficits were fewer completed categories, increased perseveration, and impaired maintenance of set on the Wisconsin Card Sorting Task (WCST), a widely used measure of executive control and cognitive flexibility. Grant et al. (2001) concluded that cognitive deficits are most likely to characterize elderly depressed people and severely depressed inpatients with psychotic features (see Harvey et al., 2004, and Rose \& Ebmeier, 2006, for similar conclusions). Tests like the WCST generally provide good sensitivity in detecting impairments in one or more components of executive control, but lack specificity. Consequently, it is unclear which processes are most likely to be affected by depression (Jonides \& Nee, 2005).

Although studies examining depression-associated deficits in the processing of nonemotional material have produced inconsistent findings, recent investigations have provided evidence of a deficit in the processing of emotional stimuli. Researchers have examined associations among inhibition of emotional material, depression, and rumination by using a modified negative priming task (Goeleven, De Raedt, Baert, \& Koster, 2006; Joormann, 2004). In this task, participants are instructed to respond to a target stimulus while ignoring a simultaneously presented emotional stimulus, clearly marked to be ignored, and irrelevant to the task; on the subsequent trial, the tobe-ignored emotional stimulus may become the target. Inhibition is operationalized as the differential delay between responding to a previously ignored stimulus and responding to a novel stimulus (Hasher et al., 1999; Tipper, 2001; Wentura, 1999). Joormann (2006) found that participants who scored high on a self-report measure of rumination exhibited a reduced ability to inhibit the processing of emotional distractors, a finding that remained significant even after controlling for level of depressive symptoms. Joormann (2004) also demonstrated that dysphoric participants and participants with a history of depressive episodes exhibit reduced inhibition of negative material that they were instructed to ignore. Finally, Goeleven et al. recently replicated these findings using a negative priming task with emotional faces. These investigators demonstrated that, in comparison with nondepressed controls, depressed participants showed impaired inhibition of sad facial expressions but intact inhibition of happy expressions. It is important to note, however, that negative priming tasks assess only one aspect of executive control - that 
is, the ability to control the insertion of relevant and irrelevant material into short-term memory, not the ability to rid one's short-term memory of irrelevant material.

Although the results of these experiments suggest that depression, and probably also rumination, involve difficulties keeping irrelevant emotional information from entering short-term memory, few studies have examined whether depression and rumination are also associated with difficulties removing previously relevant negative material from short-term memory. Importantly, no study to date has compared depressed and nondepressed participants' performance in both aspects of interference resolution within the same experimental task. Moreover, few studies have compared depressed and nondepressed participants' performance in the same task using emotional material and neutral material.

The present experiments were designed to test the formulation that depression is associated with a specific deficit in controlling the contents of short-term memory. We posit that depressed participants find it difficult to keep irrelevant material from entering short-term memory, and experience difficulties when they attempt to remove irrelevant material from short-term memory. These difficulties lead to the prolonged activation of negative material in short-term memory, resulting in sustained negative affect and recurring negative thoughts. Thus, we propose that the inability to remove irrelevant negative information from short-term memory is related to the tendency to respond to negative mood and events with rumination.

To test this hypothesis, in a fashion similar to Nee and Jonides (2008), we used two versions of the "ignore/ suppress" task, which was designed to assess people's ability to control the contents of short-term memory. In each trial of this task, four stimuli-words in Experiment 1 (see Figure 1), letters in Experiment 2-were presented simultaneously. Following the presentation of the stimuli, each trial consisted of an "ignore" part and a "suppress" part. In the ignore part (Phase 1), participants were instructed to memorize a subset of the four stimuli while ignoring the other stimuli. This part of each trial was followed by a recognition test, in which participants had to indicate whether a probe was a valid member of the target set (i.e., whether it was part of the subset of stimuli to be remembered). In the second part of each trial (Phase 2), the suppress phase, participants were instructed to forget half the stimuli they had memorized in the first part of the trial. Following the instruction to forget a subset of the memorized words, a probe was presented that could be a valid stimulus from the target set (i.e., a word that participants were supposed to remember), a stimulus that participants were supposed to ignore in Phase 1 of the experiment, a stimulus that participants were supposed to forget in Phase 2, or a new word that they had not seen at all on that trial (nonrecent probe). Phase 2 is similar to directed forgetting tasks; it should be kept in mind, however, that, whereas in the present experiments we investigated short-term memory, directed forgetting tasks were used to examine the processing of material in long-term memory.

Investigators who have used similar designs have found that participants take longer to reject recent probes that

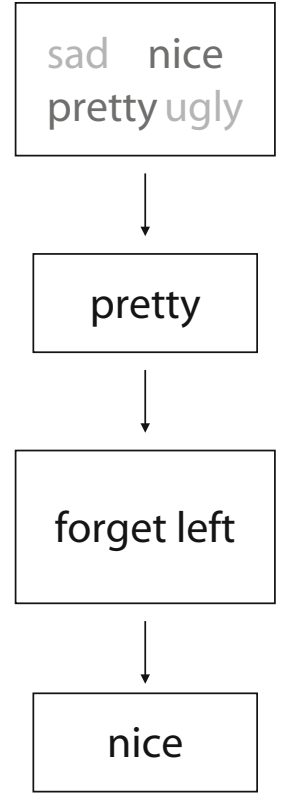

\author{
Phase 1: Ignore \\ attend to and remember \\ olive-colored words \\ $1 \mathrm{sec}$
}

Probe 1: to-be-remembered word? Yes/No

\section{Phase 2: Suppress \\ Forget cue \\ $1 \mathrm{sec}$ \\ Probe 2: to-be-remembered word? Yes/No}

Figure 1. Outline of an ignore/suppress trial.

they were instructed to ignore or to suppress (interference probes) than they do nonrecent probes (Nee \& Jonides, 2008; Nee, Jonides, \& Berman, 2007). The response latencies, therefore, assess a person's ability to stop access of irrelevant material into short-term memory (in the ignore phase) and to remove irrelevant information from short-term memory (in the suppress phase). Difficulties controlling access to short-term memory and difficulties discarding irrelevant material from working memory correspond to a greater difference in response latency when rejecting the recent probes that were to be ignored or that were to be suppressed, compared with the nonrecent probes. Given the focus of the present study on depression and rumination, in Experiment 1 we varied the valence of the words in the target and the distractor lists. To investigate whether deficits are specific to the processing of emotional material, in Experiment 2 we used an "ignore/ suppress" design with unemotional letters as stimuli. The design of Experiment 1 allowed us to compare interference probes with nonrecent probes of the same valence in order to assess participants' ability to ignore emotional material and to remove both negative and positive material from short-term memory. We predicted that, compared with their nondepressed counterparts, depressed participants would exhibit deficits in their ability both to ignore and to remove irrelevant negative material from short-term memory. We also predicted that the ability to remove irrelevant negative material from short-term memory would be related to the tendency to ruminate.

\section{EXPERIMENT 1}

\section{Method}

Overview. In both experiments, we used a version of the "ignore/ suppress" task similar to that in Nee and Jonides (2008; see Figure 1). Each trial in the experiment consisted of an ignore part and 
a suppress part. In the ignore part, participants were simultaneously presented a set of four words for $1,000 \mathrm{msec}$, half of which were presented in a light green hue and the other half in a dark green hue. Words also differed in affective valence: Either both dark green words were positive and both light green words were negative, or both dark green words were negative and both light green words were positive. Before the onset of the words, participants were instructed to attend to and remember the dark green words and to ignore the light green words. After the offset of the words and the presentation of a 1,000-msec fixation cross, participants were given a probe (Probe 1) and had to decide whether the probe matched the target set (dark green words) that they had been instructed to remember. The probe was presented in black font; it could be one of the words presented in light green (ignore), one of the words presented in dark green (remember), or a nonrecent positive or negative word. Immediately following the response, the suppress part of the task started and participants were instructed to now forget either the left or the right of the two dark green words that they had seen in the first display. These instructions were followed by the presentation of another probe (Probe 2). Participants were again instructed to indicate whether the probe matched the target word that they had been instructed to remember. Probe 2 was presented in black font; it could be the word that the participants were instructed to remember, one of the words originally presented in light green (and hence ignored), the word the participants were instructed to forget (originally presented in dark green), or a nonrecent positive or negative word. All nonrecent probes had not appeared in the previous two trials, so as to minimize familiarity with those words. Participants were asked to respond as quickly and as accurately as possible by pressing the " 1 " key on the computer keyboard for "yes" if the probe matched the target set or the " 2 " key for "no" if the probe did not match. Participants' responses and the latency of their keypresses were recorded.

Participants. Participants were solicited from two outpatient psychiatry clinics, as well as through advertisements posted in numerous locations within the community (e.g., Internet bulletin boards, university kiosks, and supermarkets). An initial phone screen established that participants were fluent in English and were between 18 and 60 years of age. Participants were excluded if they reported severe head trauma or learning disabilities, psychotic symptoms, bipolar disorder, or alcohol or substance abuse within the past 6 months. Eligible individuals were invited to come to the laboratory for a more extensive clinical interview.

The SCID (Structured Clinical Interview for the DSM-IV; First, Spitzer, Gibbon, \& Williams, 1996) has demonstrated good reliability for various disorders (Skre, Onstad, Torgersen, \& Kringlen, 1991; Williams et al., 1992). All interviewers had extensive training in the use of the SCID, as well as previous experience in administering structured clinical interviews with psychiatric patients. In previous studies, our team of interviewers achieved excellent interrater reliability of $\kappa=.93$ for the diagnosis of major depressive disorder (MDD), and $\kappa=.92$ for the "nonpsychiatric control" (CTL) diagnosis (i.e., the absence of current or lifetime psychiatric diagnoses, according to the DSM-IV criteria). Participants were included in the depressed group if they met the DSM-IV criteria for MDD. The never-disordered control group consisted of individuals with no current diagnosis and no history of any Axis I disorder. Participants were scheduled for a second session of "computer tasks," usually within 2 weeks after the interview. Forty-seven individuals (23 diagnosed with MDD and 24 never-disordered controls) participated in this study.

Questionnaires. Participants also completed the Beck Depression Inventory-II (BDI; Beck, Steer, \& Brown, 1996), a 21-item, self-report measure of the severity of depressive symptoms in this first session. We also administered the 22-item Ruminative Response Scale (RRS) of the Response Style Questionnaire (RSQ; Nolen-Hoeksema \& Morrow, 1991) to examine how participants tend to respond to sad feelings and symptoms of dysphoria. The RRS assesses responses to dysphoric mood that are focused on the self ("think about all your shortcomings, failings, faults, mistakes"), on symptoms ("think about how hard it is to concentrate"), or on possible consequences and causes of moods ("analyze recent events to try to understand why you are depressed"), using a 4-point scale (almost never to almost always). In addition, the RRS assesses behavioral responses to sad moods ("go someplace alone to think about your feelings"). Previous studies using this measure have shown good test-retest reliability and acceptable convergent and predictive validity (Nolen-Hoeksema \& Morrow, 1991; Nolen-Hoeksema, Parker, \& Larson, 1994; Treynor, Gonzalez, \& Nolen-Hoeksema, 2003). Treynor et al. suggested that the RRS is composed of two subscales that reflect adaptive and maladaptive components of rumination. Treynor et al. interpreted the 5-item reflective pondering subscales as assessing "a purposeful turning inward to engage in cognitive problem solving to alleviate one's depressive symptoms," and the 5-item brooding subscale as assessing "a passive comparison of one's current situation with some unachieved standard" (p. 256). Given that Treynor et al. report that these subscales differentially predict concurrent and future depression, we included the reflective pondering and brooding subscales in this study. Both subscales have been found to have acceptable internal consistencies and retest reliabilities (Treynor et al., 2003).

Stimuli. Words from the Affective Norms of English Words (ANEW; Bradley \& Lang, 1999) were used as stimuli. The ANEW lists valence and arousal ratings for over 1,000 English adjectives, verbs, and nouns on 9-point scales. Nouns with a rating of 4 or less were examined for possible inclusion in the negative valence condition, and nouns with a rating of 6 or more were examined for inclusion in the positive valence condition. From these lists we selected words, taking care to ensure that the positive and negative words did not differ in arousal ratings or word length. The final set of 204 positive nouns had an average valence rating of $M=7.39(S D=$ $0.55)$ and an arousal rating of $M=5.50(S D=0.88)$, whereas the final set of 204 negative nouns had an average valence rating of $M=2.71(S D=0.65)$ and an average arousal rating of $M=5.55$ $(S D=0.97)$. Positive and negative words in the two conditions did not differ on the arousal dimension or in average word length [both $t \mathrm{~s}(406)<1, \mathrm{n} . \mathrm{s}$.].

Design and Procedure. The design involved two experimentally manipulated within-subjects factors and a quasi-experimental between-subjects factor (MDD, CTL). The two within-subjects factors were the valence of the words in the ignore and suppress conditions (positive or negative) and the probe type (match probes [i.e., words presented in dark green in the ignore phase, or words that were to be remembered in the suppress phase]; interference probes [i.e., words presented in light green and ignored in the ignore phase, or words participants were instructed to forget in the suppress phase]; and nonrecent probes). Each condition was presented at least 14 times in each block (ignore phase, match and interference conditions presented 18 times, nonrecent probes presented 28 times; suppress phase, match words presented 22 times, each interference condition 14 times, nonrecent probes 14 times), and each run was composed of four blocks. After each block, participants were given feedback on their performance. After familiarizing themselves with the procedure, participants were presented with the 256 trials in four blocks with short breaks between successive blocks. The entire task lasted about $45 \mathrm{~min}$. Each trial exhibited positive and negative words: Either both dark green words were positive and both light green words were negative, or both dark green words were negative and both light green words were positive. The sequence of trials within blocks and the order of the blocks were randomized. For each participant, a random sample of words was selected from the word lists. The assignment of the color to the positive or negative list was counterbalanced.

The experiment began with a thorough explanation of the instructions to familiarize participants with the two parts of the task. Participants were instructed to respond only to the dark green words and to ignore the light green words. Each of the trials began with the presentation of a fixation cross for $1,000 \mathrm{msec}$, followed by the simultaneous presentation of four words arranged in a square. One of the words presented in the upper portion and one of the words 
Table 1

Mean Characteristics of Participants, With Standard Deviations

\begin{tabular}{|c|c|c|c|c|c|c|c|c|}
\hline & \multicolumn{4}{|c|}{ Experiment 1} & \multicolumn{4}{|c|}{ Experiment 2} \\
\hline & \multicolumn{2}{|c|}{ MDD } & \multicolumn{2}{|c|}{ CTL } & \multicolumn{2}{|c|}{ MDD } & \multicolumn{2}{|c|}{ CTL } \\
\hline & $M$ & $S D$ & $M$ & $S D$ & $M$ & $S D$ & $M$ & $S D$ \\
\hline$N(n$ female $)$ & \multicolumn{2}{|c|}{$23(17)$} & \multicolumn{2}{|c|}{$24(15)$} & \multicolumn{2}{|c|}{$21(12)$} & \multicolumn{2}{|c|}{$18(13)$} \\
\hline Age & $39.00_{\mathrm{a}}$ & 11.02 & $34.96_{\mathrm{a}}$ & 11.41 & $40.09_{\mathrm{a}}$ & 9.67 & $37.50_{\mathrm{a}}$ & 9.37 \\
\hline Years of education & $15.56_{\mathrm{a}}^{a}$ & 2.37 & $15.75_{\mathrm{a}}^{a}$ & 2.17 & $14.91_{\mathrm{a}}{ }^{a}$ & 2.81 & $15.94_{\mathrm{a}}^{a}$ & 2.94 \\
\hline BDI & $29.68_{\mathrm{a}}$ & 12.19 & $3.04_{b}$ & 7.49 & $30.51_{\mathrm{a}}$ & 8.22 & $1.61_{\mathrm{b}}$ & 2.77 \\
\hline RRS & $59.15_{\mathrm{a}}$ & 12.61 & $31.28_{\mathrm{b}}$ & 10.65 & $59.81_{\mathrm{a}}$ & 13.77 & $30.51_{\mathrm{b}}$ & 8.22 \\
\hline $\begin{array}{l}\text { Number of participants } \\
\text { with a comorbid diagnosis }\end{array}$ & \multicolumn{4}{|c|}{0} & \multicolumn{2}{|c|}{9} & \multicolumn{2}{|c|}{0} \\
\hline
\end{tabular}

Note-MDD, participants diagnosed with major depressive disorder; CTL, control group; BDI, Beck Depression Inventory;

RRS, Ruminative Response Scale. Means with the same subscript are not significantly different at $p<.05$.

presented in the lower portion were in dark green; the other words were in light green. The presentation time for this display was $1 \mathrm{sec}$. Next, another fixation cross was presented for $1 \mathrm{sec}$, followed by the first probe display. The probe display was presented until a response was detected, with a maximum duration of $3.5 \mathrm{sec}$. The first probe display was followed by another fixation cross for $1 \mathrm{sec}$, after which the "forget" cue ("forget left" or "forget right") was presented on the next slide. The "forget" cue was presented for $1 \mathrm{sec}$ and followed by another fixation cross for $1 \mathrm{sec}$. Finally, the second probe display was presented until a response was detected, or until $3.5 \mathrm{sec}$ had elapsed.

\section{Results}

Participant characteristics. Demographic and clinical characteristics of the two groups of participants are presented in Table 1. As is evident from the table, the groups did not differ significantly in age $[t(45)=1.23$, $p>.05]$ or education $[t(45)<1$, n.s.]. Unsurprisingly, the groups did differ in their BDI scores $[t(45)=13.42$, $p<.01]$. In addition, compared with the CTL participants, the MDD participants reported a greater tendency to respond to negative life events and negative mood states with rumination, as indicated by their elevated RRS scores $[t(45)=8.19, p<.01]$. Five participants in the MDD group reported current comorbid disorders, including specific phobia, social phobia, obsessive-compulsive disorder, and posttraumatic stress disorder. ${ }^{2}$ In addition, 10 MDD participants were currently taking psychotropic medication.

Interference resolution task. The task allows us to calculate various indices. In all analyses, we first examine error rates and response latencies for match probes - that is, words that participants were instructed to remember. Second, to test our main hypotheses, we calculate three interference effects (see Table 2): (1) responses to interference probes - that is, words that participants were instructed to ignore compared with nonrecent probes of the same valence presented in the ignore phase of the task (interference probes); (2) responses to interference probes compared with nonrecent probes of the same valence presented in the suppress phase of the task (interference ignore probes); and (3) responses to interference probes that people were instructed to forget compared with nonre-

Table 2

Experimental Conditions, Standard Deviations, Percentages of Error (PEs), and Response Latencies (in Milliseconds) in Experiment 1

\begin{tabular}{|c|c|c|c|c|c|c|c|}
\hline \multirow{2}{*}{$\begin{array}{c}\text { Probe } \\
\text { Valence }\end{array}$} & \multirow[b]{2}{*}{ Probe Type } & \multicolumn{3}{|c|}{ MDD } & \multicolumn{3}{|c|}{ CTL } \\
\hline & & $M$ & $S D$ & $\mathrm{PE}$ & $M$ & $S D$ & $\mathrm{PE}$ \\
\hline \multicolumn{8}{|c|}{ Ignore Condition } \\
\hline Positive & Match & 919 & 116 & 9 & 768 & 172 & 9 \\
\hline Positive & Interference & 816 & 166 & 3 & 675 & 144 & 4 \\
\hline Positive & Nonrecent & 807 & 15 & & 676 & 147 & 1 \\
\hline Negative & Match & 942 & 154 & 8 & 771 & 179 & 8 \\
\hline Negative & Interference & 828 & 165 & 4 & 677 & 137 & 4 \\
\hline Negative & Nonrecent & 817 & 156 & 2 & 666 & 121 & 1 \\
\hline \multicolumn{8}{|c|}{ Suppress Condition } \\
\hline Positive & Match & 749 & 237 & 14 & 581 & 245 & 12 \\
\hline Positive & Interference ignore & 744 & 172 & 4 & 593 & 153 & 3 \\
\hline Positive & Interference forget & 935 & 242 & 21 & 702 & 192 & 13 \\
\hline Positive & Nonrecent & 779 & 192 & 2 & 603 & 133 & 2 \\
\hline Negative & Match & 752 & 196 & 14 & 610 & 370 & 11 \\
\hline Negative & Interference ignore & 760 & 168 & 4 & 600 & 139 & 4 \\
\hline Negative & Interference forget & 975 & 257 & 19 & 683 & 248 & 11 \\
\hline Negative & Nonrecent & 724 & 162 & 2 & 618 & 149 & 2 \\
\hline
\end{tabular}

Note-MDD, participants diagnosed with major depressive disorder; CTL, control group. 
cent probes of the same valence presented in the suppress phase of the task (interference forget probes). We evaluate group and valence effects on these four indices by using separate mixed-effects ANOVAs with group as a betweensubjects factor and probe valence of the words and probe type as within-subjects factors.

Error responses. The mean percentages of error responses for the different probes are presented in Table 2. Overall, error rates were low (MDD, 6.5\%; CTL, 4.9\%). We conducted mixed effects ANOVAs to examine differences in the percentages of error responses as a function of group, valence, and probe type. For responses to match probes in the ignore and suppress phases of the task, we found no main effects of group or valence and no interactions [all $F \mathrm{~s}(1,45)<1]$. We then conducted three-way group (MDD, $\mathrm{CTL}) \times$ probe type (interference, nonrecent $) \times$ probe valence (positive, negative) ANOVAs to investigate interference effects in the ignore and suppress phases. In these ANOVAs, we obtained main effects of condition [ignore phase, $F(1,45)=7.26, p<.01$; suppress phase, $F(1,45)=$ $19.16, p<.01$, for probes participants were instructed to ignore in the ignore phase; and $F(1,45)=33.54, p<.01$, for forget probes]. No other significant main effects or interactions were obtained. In sum, all participants made fewer errors when evaluating a nonrecent probe than when doing so with an interference probe. No differences between depressed and control participants emerged for error rates in any of our comparisons.

Decision latencies to match probes. We restricted our analyses of decision latencies to trials on which participants made correct responses. To eliminate outliers, decision latencies that exceeded $1.5 \mathrm{sec}$ were treated as missing values: less than $8 \%$ of all reaction times (RTs). No group differences in the number of outlying latencies were obtained $[F(1,45)<1]$. Mean decision latencies for participants in the two groups are also presented in Table 2. We had no specific predictions for group or valence differences in response to the match probes. Two-way group $(\mathrm{MDD}, \mathrm{CTL}) \times$ probe valence (positive, negative) ANOVAs conducted on the decision latencies in response to match probes yielded only significant main effects for group: In both the ignore $[F(1,45)=12.47$, $p<.01]$ and suppress $[F(1,45)=4.20, p<.05]$ phases of the task, MDD participants took longer to respond to the match probes than did CTL participants.

Decision latencies to interference probes (interference effects). Our main hypotheses involved decision latencies to the interference probes. If depressed participants have trouble ignoring negative material or discarding negative material from working memory, they should take longer to reject negative interference probes than to reject nonrecent probes of the same valence. Thus, we predicted a significant three-way interaction of group, valence, and probe type for interference probes in the ignore phase and for interference ignore and forget probes in the suppress phase of the task. We expected that depressed participants would show difficulty both in ignoring irrelevant negative material (negative ignore probes vs. negative nonrecent probes) and in removing irrelevant negative words from short-term memory (negative forget probes vs. negative nonrecent probes). Thus, MDD participants should be significantly slower than controls to decide whether recent negative interference probes matched the target set. No group differences were expected for decisions about nonrecent probes of the same valence. We tested these predictions by analyzing decision latencies in the ignore and suppress phases of the task. In the ignore phase of the task, a three-way group $(\mathrm{MDD}, \mathrm{CTL}) \times$ probe valence (positive, negative) $\times$ probe type (interference, nonrecent) mixed effects ANOVA yielded only a significant main effect for group $[F(1,45)=11.37, p<.01]$.

For the suppress phase, we first analyzed RTs to probes that participants were supposed to ignore in the first phase of the task, using a three-way group $(\mathrm{MDD}, \mathrm{CTL}) \times$ probe valence (positive, negative) $\times$ probe type (interference ignore, nonrecent) mixed effects ANOVA. This analysis yielded a significant main effect for group $[F(1,45)=$ $10.81, p<.01]$ and a trend for a valence $\times$ probe type interaction $[F(1,45)=2.87, p<.10]$; no other main effects or interactions were significant. We tested the hypothesis that MDD participants experienced difficulty removing irrelevant material from short-term memory by using a three-way group $(\mathrm{MDD}, \mathrm{CTL}) \times$ probe valence (positive, negative) $\times$ probe type (interference forget, nonrecent) mixed effects ANOVA to analyze response latencies to the forget probes. This analysis yielded a main effect for group $[F(1,45)=14.73, p<.01]$, a main effect for probe type $[F(1,45)=49.96, p<.01]$, an interaction of probe type and group $[F(1,45)=4.05, p<.01]$, and the predicted significant three-way interaction of group, probe type, and valence $[F(1,45)=7.87, p<.01] .^{3}$

To examine this interaction further, we calculated interference effects (decision latencies to interference forget probes minus decision latencies to nonrecent probes of the same valence), which are presented in Figure 2. No group differences were found in interference effects when comparing responses to positive material [all $t \mathrm{~s}(45)<1$, n.s.]. As predicted, however, MDD participants had significantly greater interference effects than did the CTL participants when processing negative material $[t(45)=$ 4.01, $p<.01$, ES: $d=1.17]$.

To summarize, we did not find interference effects of ignored material or differences in interference of ignored material as a function of group, although there was a trend toward an interaction of interference and valence. By contrast, interference from material suppressed from short-term memory showed the predicted three-way interaction, with both groups demonstrating significant interference, and depressed participants showing greater interference, especially when probed with discarded negative information.

Interference effects and rumination. Our second hypothesis was that interference effects would be correlated significantly with individual differences in rumination. These correlations are presented in Table 3. As predicted, interference effects due to suppression of negative material were significantly correlated with rumination; moreover, individual differences in interference explained a signifi- 


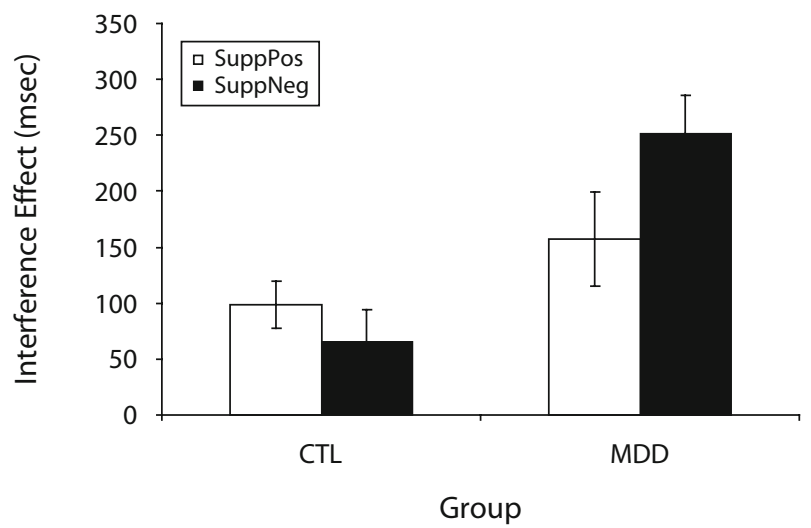

Figure 2. Experiment 1: Mean interference effects for negative and positive probes in the suppress condition (reaction time [RT] to forget probes - RT to nonrecent probes) in participants diagnosed with major depressive disorder (MDD) and control participants (CTL). Error bars represent one standard error.

cant part of the variance in the rumination scores, even after controlling for individual differences in interference effects for positive material. These results indicate that it is not so much a general deficit in removing irrelevant material from short-term memory that is a problem for depressed individuals, but rather a specific difficulty in removing irrelevant negative material related to rumination.

\section{EXPERIMENT 2}

To further investigate whether our findings reflect a specific deficit in removing negative material from shortterm memory versus a more general inhibitory deficit, we used single letters instead of emotional words in the ignore/suppress task in Experiment 2. That is, we used material that has no obvious valence. As outlined previously, research on general cognitive deficits in depression has produced inconsistent findings. This may be due in part to variation in the difficulty of the tasks used in different studies. Traditionally, research on cognitive deficits in depression has suggested that the observation of depressive deficits is dependent on task difficulty. The resource-allocation hypothesis, for example, postulates that because their cognitive capacity is reduced, depressed individuals have deficits in engaging in effortful cognitive procedures (e.g., Ellis \& Ashbrook, 1988). The general assumption is that there is a limit on the resources available for cognitive operations, and that depression either occupies or functionally reduces these resources (e.g., Ellis, Thomas, \& Rodriguez, 1984; Seibert \& Ellis, 1991). Deficits should thus become evident in effortful tasks; whereas depressed participants may not differ from control participants in tasks characterized by low difficulty, group differences should be detectable in difficult tasks. Given these theoretical predictions, in addition to previous findings suggesting that depressive deficits in short-term memory tasks do indeed depend on task difficulty (e.g., Rose \& Ebmeier, 2006), we varied the memory load in this task by presenting either four or eight letters in the learning display.

\section{Method}

Participants and Questionnaires. Recruitment and selection criteria were identical to those described in Experiment 1. Twentyone MDD and 18 CTL participants were administered the ignore/ suppress task. The SCID interview was used to assign participants to these two groups, and all participants were given the BDI and the RRS. Table 1 presents the demographic information for this sample. Comorbid diagnoses in this sample were similar to those in Experiment 1; 9 MDD participants received comorbid diagnoses including panic disorder, social phobia, obsessive-compulsive disorder, and posttraumatic stress disorder. In addition, $15 \mathrm{MDD}$ participants were currently taking psychotropic medication.

Design and Procedure. The ignore/suppress task was identical to the task described in Experiment 1, with the exception that letters were presented instead of words. In addition, half of the trials presented four letters and half presented eight letters. Participants were presented with blocks of four-letter displays and blocks of eight-letter displays; the order of the four- and eight-letter blocks was counterbalanced. The design therefore involved two experimentally manipulated within-subjects factors and a quasi-experimental between-subjects factor (MDD, CTL). The within-subjects factors were the load (four vs. eight letters) and the probe type (match probes - i.e., letters presented in dark green or letters that were to be remembered in the suppress phase; interference probes-i.e., letters presented in light green and ignored, or letters participants were instructed to forget; and nonrecent probes). Each condition was presented at least 14 times in each block, and each run was composed of eight blocks. After each block, participants were given feedback on their performance. After familiarizing themselves with the procedure and the stimuli, participants were presented with the 512 trials in eight blocks, with short breaks between blocks. The entire task took about $45 \mathrm{~min}$.

The experiment began with a thorough explanation of the instructions to familiarize participants with the two parts of the task. Participants were instructed to encode and remember the dark green letters and to ignore the light green letters. Each trial began with the presentation of a fixation cross for $1,000 \mathrm{msec}$, followed by the simultaneous presentation of four or eight letters arranged in two rows. In a manner similar to Experiment 1 (see Figure 1), half of the letters were presented in light green (one in each row) and half were presented in dark green. The presentation time for this display was $1 \mathrm{sec}$, and it was followed by a 500 -msec mask. Next, another fixation cross was presented for $1 \mathrm{sec}$, followed by the first probe display. The probe display was presented until a response was detected but for a maximum of $3.5 \mathrm{sec}$. The first probe display was followed by another fixation cross for $1 \mathrm{sec}$, after which the forget cue (forget left or forget right) was presented on the next screen. The forget cue was presented for $1 \mathrm{sec}$ and followed by another fixation cross for $1 \mathrm{sec}$. Finally, the second probe display was presented until a response was detected or $3.5 \mathrm{sec}$ had elapsed.

\section{Results}

Error responses. The mean percentages of error responses are presented in Table 4. Overall, error rates were

Table 3

Correlations and Regression Analysis of Interference Effects, Beck Depression Inventory (BDI) Scores, and Rumination

\begin{tabular}{lcrrrr}
\hline Measures & NegInt & PosInt & DV: RRS & $\beta$ & $R^{2}$ \\
\hline BDI & $.50^{*}$ & .15 & Step 1: PosInt & -.13 & .02 \\
RRS & $.41^{*}$ & .04 & Step 2: PosInt & $-.40^{*}$ & $.21^{*}$ \\
Reflection & $.31^{*}$ & -.13 & NegInt & $.52^{*}$ & \\
Brooding & .08 & -.14 & & & \\
\hline
\end{tabular}

Note $-N=47$. NegInt, interference effect for negative material in the ignore/suppress task; PosInt, interference effect for positive material in the ignore/suppress task; RRS, Ruminative Response Scale; Reflection/ Brooding, subscales of the RRS. ${ }^{*} p<.05$. 
low (MDD, 8.6\%; CTL, 5.4\%). We conducted mixed effects ANOVAs to examine differences in the percentages of error responses as a function of group and experimental condition. For responses in the ignore phase of the task, we first conducted a two-way group $(\mathrm{MDD}, \mathrm{CTL}) \times$ load (four or eight letters) ANOVA to compare responses to the match probes. This analysis yielded a significant main effect of load $[F(1,37)=15.79, p<.01]$ and a significant interaction of load and group $[F(1,37)=6.38, p<$ $.01]$, but no main effect of group and no other interactions $[F \mathrm{~s}(1,37)<1]$. Follow-up tests showed a trend toward more errors in the MDD than in the CTL group under low load $[t(37)=1.90, p<.06]$, but not under high load $[t(37)<1$, n.s.]. We conducted a three-way group (MDD, $\mathrm{CTL}) \times$ probe type (interference, nonrecent $) \times$ load (four vs. eight letters) ANOVA to investigate interference effects in the ignore phase. This ANOVA yielded significant main effects for load $[F(1,37)=6.26, p<.02]$ and probe type $[F(1,37)=7.33, p<.01]$, which were qualified by an interaction of probe type and load $[F(1,37)=12.61$, $p<.01]$. This interaction was due to stronger interference effects for all participants in the high-load condition. No main effect of group and no interactions with group were significant.

For the suppress phase of the task, we first conducted a two-way group $(\mathrm{MDD}, \mathrm{CTL}) \times$ load (four vs. eight letters) ANOVA to compare responses to the match probes. This analysis yielded only a significant main effect of load $[F(1,37)=9.36, p<.01]$. We also conducted a three-way group $(\mathrm{MDD}, \mathrm{CTL}) \times$ load (four vs. eight letters $) \times$ probe type (interference probe ignore, nonrecent probe) ANOVA for the suppress phase of the task. This ANOVA yielded a significant effect only for load $[F(1,37)=5.50, p<.03]$. Finally, a three-way group $($ MDD, CTL) $\times$ load (four vs. eight letters) $\times$ probe type (interference probe suppress, nonrecent probe) ANOVA conducted on the forget probes yielded only a significant effect for probe type $[F(1,37)=$ $24.15, p<.01]$. In sum, these findings show that all participants made fewer errors under decreased load. Importantly, no group main effects and no interactions of probe type and group were found. Only one reliable betweengroups difference emerged: Depressed participants made more errors than did nondepressed participants when responding to match probes in the first phase of the task, but only in the low-load (i.e., four-letter) condition.

Decision latencies to match probes. To eliminate outliers, decision latencies that exceeded $1.5 \mathrm{sec}$ were treated as missing values (less than $6 \%$ of all RTs). Only correct responses were analyzed. No group differences were obtained in the number of outlying latencies $[F(1,37)<1$, n.s.]. Mean decision latencies for participants in the two groups are presented in Table 4. We had no specific predictions for group differences in response to the match probes. Two-way group (MDD, CTL) $\times$ load (four vs. eight letters) ANOVAs conducted on the decision latencies in response to match probes in the ignore and suppress phases of the task yielded significant main effects for load, which were qualified by significant interactions of load and group. In both the ignore $[F(1,37)=8.15, p<$ $.01]$ and the suppress $[F(1,37)=8.18, p<.01]$ phase, MDD participants were slower to respond to match probes under low load but did not differ from CTL participants under high load.

Decision latencies to interference probes (interference effects). Our main hypotheses involved decision latencies to the interference probes. We analyzed response latencies in the ignore phase of the task with a threeway group $(\mathrm{MDD}, \mathrm{CTL}) \times$ load (four, eight $) \times$ probe type (interference, nonrecent) mixed effects ANOVA. This analysis yielded a significant main effect for probe type $[F(1,37)=5.42, p<.01]$, a main effect for load $[F(1,37)=85.62, p<.01]$, and an interaction of load

Table 4

Experimental Conditions, Standard Deviations, Percentages of Error (PEs), and Response Latencies (in Milliseconds) in Experiment 2

\begin{tabular}{|c|c|c|c|c|c|c|c|}
\hline \multirow[b]{2}{*}{ Letters } & \multirow[b]{2}{*}{ Probe Type } & \multicolumn{3}{|c|}{ MDD } & \multicolumn{3}{|c|}{ CTL } \\
\hline & & $M$ & $S D$ & $\mathrm{PE}$ & $M$ & $S D$ & $\mathrm{PE}$ \\
\hline \multicolumn{8}{|c|}{ Ignore Condition } \\
\hline 4 & Match & 702 & 210 & 15 & 583 & 139 & 6 \\
\hline 4 & Interference & 866 & 170 & 4 & 765 & 151 & 2 \\
\hline 4 & Nonrecent & 853 & 165 & 5 & 762 & 148 & 1 \\
\hline 8 & Match & 793 & 191 & 17 & 755 & 177 & 14 \\
\hline 8 & Interference & 932 & 161 & 7 & 894 & 140 & 5 \\
\hline 8 & Nonrecent & 915 & 158 & 5 & 874 & 145 & 3 \\
\hline \multicolumn{8}{|c|}{ Suppress Condition } \\
\hline 4 & Match & 861 & 141 & 5 & 805 & 142 & 2 \\
\hline 4 & Interference ignore & 715 & 173 & 3 & 637 & 129 & 1 \\
\hline 4 & Interference forget & 789 & 158 & 19 & 724 & 159 & 12 \\
\hline 4 & Nonrecent & 719 & 163 & 4 & 648 & 148 & 1 \\
\hline 8 & Match & 932 & 131 & 9 & 922 & 139 & 6 \\
\hline 8 & Interference ignore & 806 & 199 & 5 & 762 & 147 & 4 \\
\hline 8 & Interference forget & 899 & 166 & 18 & 843 & 168 & 17 \\
\hline 8 & Nonrecent & 785 & 162 & 7 & 752 & 165 & 2 \\
\hline
\end{tabular}

Note-MDD, participants diagnosed with major depressive disorder; CTL, control group. 
and group $[F(1,37)=7.84, p<.01]$; there was no main effect for group and no interaction of group and probe type. The interaction of load and group was due to CTL participants exhibiting a greater RT difference than did MDD participants for low- versus high-load conditions. For the suppress phase, we first analyzed RTs to probes that participants were supposed to ignore in the first part of the task. A three-way group (MDD, CTL) $\times$ load (four, eight) $\times$ probe type (interference ignore, nonrecent) mixed-effects ANOVA yielded only a significant main effect for load $[F(1,37)=86.24, p<.01]$. Finally, by analyzing response latencies to the forget probes, we tested the hypothesis that MDD participants experience difficulties removing irrelevant material from short-term memory. A three-way group $(\mathrm{MDD}, \mathrm{CTL}) \times$ load (four, eight) $\times$ probe type (interference suppress, nonrecent) mixed effects ANOVA yielded main effects for load $[F(1,37)=73.91$, $p<.01]$ and probe type $[F(1,37)=71.22, p<.01]$, but no main effect for group and no interactions with group. To compare the findings in Experiment 2 with the findings in Experiment 1, we calculated interference effects (decision latencies to interference forget probes minus decision latencies to control probes). No group differences were obtained for interference effects [four letters, $t(37)<1$; $\mathrm{ES}, d=0.08$; eight letters, $t(37)<1$; ES, $d=0.24]$. No significant correlations were found between interference effects in this task and BDI [four letters, $r(39)=-.04$; eight letters, $r(39)=.01$ ] or RRS scores [four letters, $r(39)=.11$; eight letters, $r(39)=.18$ ]. In sum, although load led to slower RTs in all participants, and although we found strong evidence for interference effects in MDD and CTL participants in the letter version of the task, thus replicating previous results obtained using this task in an unselected sample (e.g., Nee \& Jonides, 2008), we did not obtain group differences in interference effects when using letters instead of emotional words. In addition, load did not affect group differences in interference. We did find, however, that compared with their nondepressed counterparts, MDD participants made more errors and exhibited longer RTs to match probes when under lowload but not high load conditions.

\section{DISCUSSION}

Depression is associated with a tendency to respond to negative mood states and negative life events with ruminative thinking (Nolen-Hoeksema, 2000; Nolen-Hoeksema et al., 2008). Rumination, in turn, has been linked to prolonged negative affect and to a heightened vulnerability for the onset and maintenance of depressive episodes (Lyubomirsky \& Nolen-Hoeksema, 1993; Nolen-Hoeksema, Morrow, \& Fredrickson, 1993). Despite this growing body of research, however, it is still unclear why some people are especially prone to rumination, whereas others find it relatively easy to reorient and recover from sad mood states. The present study was designed to use an emotional and a nonemotional version of the ignore/suppress task to test the hypotheses that depressed individuals experience difficulties in cognitive control and that these difficulties are associated with an increased tendency to ruminate.
Surprisingly, the results of this study indicate that participants diagnosed with MDD do not differ from control participants in their ability to ignore emotional material. Regardless of whether we presented positive or negative material, or material without any obvious valence (i.e., single letters), depressed and nondepressed participants exhibited similar interference effects (or lack thereof) when responding to a probe that they had previously been instructed to ignore. Furthermore, it did not matter whether interference from the to-be-ignored material was tested immediately after participants had been given the instruction to ignore it, or later in the task; neither case produced group differences. These findings suggest that depressed participants can stop entry of irrelevant material into short-term memory as effectively as nondepressed controls can.

However, we did find that depressed participants exhibited difficulties removing irrelevant negative material from short-term memory. Specifically, in comparison with never-depressed controls, depressed individuals exhibited greater decision latencies to an interference probe (i.e., a probe they were supposed to forget) than to a nonrecent probe, reflecting the strength of the residual activation of the contents of short-term memory that were declared to be no longer relevant. Importantly, this pattern was not found for positive material. Moreover, the ability to remove irrelevant material from short-term memory was correlated with individual differences in rumination, further strengthening the proposition that deficits in interference resolution underlie the tendency to ruminate. To examine whether these difficulties were due to a general processing deficit, we also compared the performance of depressed and control participants on a version of the same task that used neutral stimuli (e.g., single letters) instead of emotional words. We found that depressed and nondepressed participants did not differ in the strength of interference created by recent to-be-forgotten probes on this task, providing further evidence for the proposition that our findings in Experiment 1 were not due to a general deficit in cognitive control. In sum, the present findings indicate that depression and rumination are associated with increased interference from irrelevant negative material—specifically, with difficulties in removing irrelevant negative material from short-term memory.

This study adds to a small but growing literature linking depression and rumination with deficits in executive control. Most of these studies have employed tasks that rely on a variety of executive processes, making it difficult to specify exactly what mechanisms are impaired in depression and rumination. For example, Davis and NolenHoeksema (2000) used the Wisconsin Card Sorting Task and found that, compared with nonruminators, ruminators made more perseverative errors, regardless of their level of depressive symptomatology. Watkins and Brown (2002) induced rumination in depressed participants and demonstrated that these individuals showed stereotyped counting responses in a random-number generating task, reflecting their difficulty inhibiting prepotent responses. Recently, Whitmer and Banich (2007) employed a taskswitching design and demonstrated that in a student 
sample self-reported rumination was not associated with difficulties in switching to a new set but with difficulties in inhibiting prior mental sets. Interestingly, our findings suggest that neither the ability to ignore irrelevant material nor the ability to remove irrelevant material from short-term memory is impaired in depression when the materials are nonemotional. In addition, we found that the reflection, but not the brooding, subcomponent of rumination was significantly correlated with interference. This is surprising, given that previous studies have characterized reflection as a more adaptive form of rumination (Treynor et al., 2003). It should be noted, however, that studies examining rumination in clinical samples have reported results indicating that, for diagnosed samples of depressed participants, this "adaptive" form of rumination may actually be problematic (Joormann, Dkane, \& Gotlib, 2006). Future research that focuses on identifying the specific executive processes affected in depression and underlying rumination will be critical in improving our understanding of this disorder and in developing interventions that target these processes.

It is noteworthy that differences between MDD and CTL participants were obtained only for negative material. It is not clear, however, whether this represents a valence-specific deficit in executive control, or whether stronger activation associated with negative material in depression makes it more difficult to control processing, even in the absence of deficits in executive control. It is also important to note that two studies that have used a directed forgetting paradigm to examine intentional removal of items from long-term memory report similar valence-specific effects in depressed participants (Power, Dalgleish, Claudio, Tata, \& Kentish, 2000) and in unselected samples under a negative mood induction (Minnema \& Knowlton, 2008) and, further, that both studies attribute their findings to a disruption in cognitive control processes by negative emotion. Clearly, future research is needed to gain a better understanding of the nature of these deficits in executive control in depression.

Although no group differences were obtained for interference resolution in Experiment 2, an interesting effect of load was obtained in response to match probes. As discussed earlier, resource allocation models predict that depressive deficits should be detectable primarily under conditions of high cognitive load. In contrast to this prediction, depressed participants made more errors and took longer to make decisions when confronted with match probes under conditions of low cognitive load. Depressed participants, however, did not differ from control participants under conditions of high load. These findings suggest that there is no linear relation between task difficulty and depressive deficits; indeed, they suggest that depressive deficits are more readily observed when cognitive load is low. Hertel (1998) has provided intriguing data that suggests that "easy tasks"- that is, tasks that do not require participants to fully engage with the task and that do not control participants' attention-are more likely to provide evidence for depressive deficits. According to Hertel (2004), these tasks permit rumination and mind wandering and, therefore, are sensitive to individual differences in cognitive control and in the use of effective strategies. Although these are interesting points for further discussion, given that we did not predict this pattern of findings these results clearly await replication.

Given that the majority of previous studies only assessed executive functions while participants were processing neutral stimuli, they do not address the important question of why rumination typically involves negatively valenced material. Noteworthy exceptions are recent studies in which investigators have used a negative affective priming task to examine inhibition of emotional stimuli in depression. Although the results of these studies indicate that current depression and a lifetime diagnosis of depressive episodes are related to decreased inhibition in the processing of negative material (Goeleven et al., 2006; Joormann, 2004), the present study is the first to compare depression-related differences in perceptual processing of emotional material with differences in expelling material from short-term memory within the same task. In contrast to these earlier studies, we did not obtain evidence for a depressive deficit in ignoring negative material. It is important to keep in mind, though, that in the negative priming designs, participants are instructed to evaluate the affective valence of the presented stimuli; in the ignore/ suppress task, participants are instructed to remember the stimuli. Thus, it is possible that depression is not associated with deficits in perceptual processing, but that it is difficult for depressed participants to ignore negative material once they start processing the semantic content or affective valence of this material. The short presentation duration of materials used here may have further reduced the appreciation of the semantic or affective valence of to-be-ignored material. Future studies should investigate this formulation more explicitly by systematically varying task instructions and presentation durations.

It is also interesting that Goeleven et al. (2006) found that self-reported level of rumination was not related to inhibition of sad faces in a negative affective priming task. Goeleven et al. suggested that this finding might be due to the use of facial expressions, underscoring the potentially important association between rumination and verbal material, such as that found in the present study. In addition, however, it is possible that rumination is related more closely to difficulties expelling negative material from short-term memory than it is to difficulties controlling access of negative material to short-term memory. Indeed, in a recent study, Joormann and Gotlib (2008) used a design that focused only on individual differences in updating the content of short-term memory and found that participants diagnosed with MDD had trouble expelling negative material. In addition, difficulties in expelling negative material were correlated with self-reported rumination. The present study adds to this line of research by demonstrating that within the same task, no evidence is obtained for group differences in ignoring negative material, but clear support is obtained for group differences in removing negative material from short-term memory. The present study also demonstrates that no such performance pattern emerges when depressed participants process nonemotional material, suggesting that depression 
and rumination are associated with deficits in a specific aspect of executive control.

Given the cross-sectional design of the present study, formulations concerning underlying mechanisms and consequences are necessarily speculative. Although investigators have demonstrated that rumination is not simply a symptom of depression, but that it also predicts the onset of depressive episodes (Just \& Alloy, 1997; Nolen-Hoeksema, 2000), the specific role that interference resolution may play in this association is unclear. Longitudinal studies are needed to assess individual differences in these processes prior to the onset of rumination and/or depression in order to provide clear evidence that interference resolution deficits underlie rumination and thereby increase the risk for the onset and maintenance of depressive episodes. Future studies should include additional measures of rumination and, ideally, should compare self-reported rumination and experimental rumination manipulations.

It is also important to keep in mind that the MDD and CTL groups differed in overall RT. Although this finding is not unexpected, it is possible that this effect led to an overestimation of group differences (Faust, Balota, Spieler, \& Ferraro, 1999). Given our finding of a group difference in the removal of irrelevant negative but not of positive stimuli from short-term memory, however, we do not think that this is a likely explanation for our findings. Moreover, we redid our analyses using proportion RT scores and replicated our findings (see note 3 ). We should also note that neither MDDs nor CTLs exhibited interference effects in the first part of the task in Experiment 1 . It is possible, therefore, that differences between the ignore and suppress parts in Experiment 1 are due to differential sensitivity of the two parts of this task in detecting group differences. Nevertheless, the comparison of two different aspects of short-term memory functioning within the same task using the same group of participants represents an important improvement over previous work that has compared these processes across different studies or across different tasks. As we outlined above, future research should systematically vary the encoding instructions and presentation durations to examine whether the obtained differences are observed only in semantic processing and not in perceptual processing. Given the already lengthy protocol, it was not feasible to include positive, negative, and neutral material within the same study. It is possible, therefore, that our lack of findings in Experiment 2 is not due to the use of neutral material but, rather, to a lack of power. Given the effect size for the group difference in Experiment 1, however, power analyses indicate that Experiment 2 had a power of .97 to detect an effect of similar size, which renders this explanation unlikely. Nevertheless, it will be important in future research to compare emotional and neutral material within the same task.

In sum, the present study is important as a beginning in the process of elucidating the nature of the relations linking rumination, executive control, and depression. Given that the experience of negative mood states and negative life events is associated with the activation of mood-congruent cognitions in short-term memory, the ability to control the contents of short-term memory may be crucial in understanding individual differences in emotional regulation. The ability to expel mood-congruent material from shortterm memory may help us differentiate people who recover easily from negative affect from those who initiate a vicious circle of increasingly negative ruminative thinking and deepening sad mood. Investigating individual differences in executive functions, therefore, has the potential to provide important insights into the maintenance of negative affect and vulnerability to experience depressive episodes.

\section{AUTHOR NOTE}

This research was supported by National Institute of Mental Health Grants MH59259 and MH60655 awarded to I. H. Gotlib and J. Jonides, respectively. In addition, support for M.G.B. and D.E.N. was provided by National Science Foundation Graduate Fellowships. Correspondence concerning this article should be addressed to J. Joormann, Department of Psychology, University of Miami, P.O. Box 248185, Coral Gables, FL 33124-0751 (e-mail: j.joormann@miami.edu).

\section{REFERENCES}

Barch, D. M., Sheline, Y. I., Csernansky, J. G., \& Snyder, A. Z. (2003). Working memory and prefrontal cortex dysfunction: Specificity to schizophrenia compared with major depression. Biological Psychiatry, 53, 376-384.

Beck, A. T., Steer, R. A., \& Brown, G. K. (1996). Manual for the Beck Depression Inventory-II. San Antonio: Psychological Corporation.

BJorklund, D. F., \& HARnishFeger, K. K. (1990). The resources construct in cognitive development: Diverse sources of evidence and a theory of inefficient inhibition. Developmental Review, 10, 48-71.

Bradley, M. M., \& Lang, P. J. (1999). Affective norms for English words (ANEW): Instruction manual and affective ratings (Tech. Rep. C-1). Gainesville: University of Florida, Center for Research in Psychophysiology.

Channon, S., Baker, J. E., \& Robertson, M. M. (1993). Working memory in clinical depression: An experimental study. Psychological Medicine, 23, 87-91

Cowan, N. (1999). An embedded-processes model of working memory. In A. Miyake \& P. Shah (Eds.), Models of working memory: Mechanisms of active maintenance and executive control (pp. 62-101). New York: Cambridge University Press.

Davis, R. N., \& Nolen-Hoeksema, S. (2000). Cognitive inflexibility among ruminators and nonruminators. Cognitive Therapy \& Research, 24, 699-711.

Egeland, J., Rund, B. R., Sundet, K., Landrø, N. I., AsbJøRnsen, A., LUND, A., ET AL. (2003). Attention profile in schizophrenia compared with depression: Differential effects of processing speed, selective attention and vigilance. Acta Psychiatrica Scandinavica, 108, 276-284.

Ellis, H. C., \& Ashbrook, P. W. (1988). Resource allocation model of the effects of depressed mood states on memory. In K. Fiedler \& J. P. Forgas (Eds.), Affect, cognition, and social behavior (pp. 25-43). Toronto: Hogrefe.

Ellis, H. C., Thomas, R. L., \& Rodriguez, I. A. (1984). Emotional mood states and memory: Elaborative encoding, semantic processing, and cognitive effort. Journal of Experimental Psychology: Learning, Memory, \& Cognition, 10, 470-482.

Engle, R. W., KANE, M. J., \& Tuholski, S. W. (1999). Individual differences in working memory capacity and what they tell us about controlled attention, general fluid intelligence, and functions of the prefrontal cortex. In A. Miyake \& P. Shah (Eds.), Models of working memory: Mechanisms of active maintenance and executive control (pp. 102-134). Cambridge: Cambridge University Press.

EnRight, S. J., \& BeEch, A. R. (1990). Obsessional states: Anxiety disorders or schizotypes? An information processing and personality assessment. Psychological Medicine, 20, 621-627.

Erber, R., \& Erber, M. W. (1994). Beyond mood and social judgment: Mood incongruent recall and mood regulation. European Journal of Social Psychology, 24, 79-88.

Faust, M. E., Balota, D. A., Spieler, D. H., \& Ferraro, F. R. (1999). 
Individual differences in information-processing rate and amount: Implications for group differences in response latency. Psychological Bulletin, 125, 777-799.

First, M. B., Spitzer, R. L., Gibbon, M., \& Williams, J. B.W. (1996). Structured clinical interview for DSM-IV axis I disorders-clinician version $(S C I D-C V)$. Washington, DC: American Psychiatric Press.

Fossati, P., Amar, G., Raoux, N., Ergis, A., \& Allilaire, J. F. (1999). Executive functioning and verbal memory in young patients with unipolar depression and schizophrenia. Psychiatry Research, 89, 171-187.

Friedman, N. P., \& MiYaKe, A. (2004). The relations among inhibition and interference control functions: A latent-variable analysis. Journal of Experimental Psychology: General, 133, 101-135.

FrITH, C. D. (1979). Consciousness, information processing and schizophrenia. British Journal of Psychiatry, 134, 225-235.

Goeleven, E., De Raedt, R., Baert, S., \& Koster, E. H. W. (2006). Deficient inhibition of emotional information in depression. Journal of Affective Disorders, 93, 149-157.

Grant, M. M., Thase, M. E., \& Sweeney, J. A. (2001). Cognitive disturbances in outpatient depressed younger adults: Evidence of modest impairment. Biological Psychiatry, 50, 35-43.

Harvey, P. O., Le Bastard, G., Pochon, J. B., Levy, R., Allilaire, J. F., Dubois, B., \& Fossati, P. (2004). Executive functions and updating of the contents of working memory in unipolar depressions. Journal of Psychiatric Research, 38, 567-576.

Hasher, L., Stoltzfus, E. R., Zacks, R. T., \& Rypma, B. (1991). Age and inhibition. Journal of Experimental Psychology: Learning, Memory, \& Cognition, 17, 163-169.

HASHER, L., \& ZACKS, R. T. (1988). Working memory, comprehension, and aging: A review and a new view. In G. H. Bower (Ed.), The psychology of learning and motivation (Vol. 22, pp. 193-225). San Diego: Academic Press.

Hasher, L., Zacks, R. T., \& MAY, C. P. (1999). Inhibitory control, circadian arousal, and age. In D. Gopher \& A. Koriat (Eds.), Attention and performance XVII: Cognitive regulation of performance. Interaction of theory and application (pp. 653-675). Cambridge, MA: MIT Press.

Hertel, P. T. (1997). On the contributions of deficient cognitive control to memory impairments in depression. Cognition \& Emotion, 11, 569-583.

HerTEL, P. T. (1998). The relationship between rumination and impaired memory in dysphoric moods. Journal of Abnormal Psychology, 107, 166-172.

Hertel, P. T. (2004). Memory for emotional and nonemotional events in depression: A question of habit? In D. Reisberg \& P. [T.] Hertel (Eds.), Memory and emotion (pp. 186-216). New York: Oxford University Press.

IsEN, A. M. (1984). Toward understanding the role of affect in cognition. In R. S. Wyer \& T. K. Srull (Eds.), Handbook of social cognition (pp. 179-236). Hillsdale, NJ: Erlbaum.

Jonides, J., Lewis, R. L., Nee, D. E., Lustig, C. A., Berman, M. G., \& Moore, K. S. (2008). The mind and brain of short-term memory. Annual Review of Psychology, 59, 193-224.

Jonides, J., \& NeE, D. E. (2005). Assessing dysfunction using refined cognitive methods. Schizophrenia Bulletin, 31, 823-829.

Joormann, J. (2004). Attentional bias in dysphoria: The role of inhibitory processes. Cognition \& Emotion, 18, 125-147.

JOORMANN, J. (2005). Inhibition, rumination, and mood regulation in depression. In R. W. Engle, G. Sedek, U. von Hecker, \& D. N. McIntosh (Eds.), Cognitive limitations in aging and psychopathology: Attention, working memory, and executive functions (pp. 275-312). New York: Cambridge University Press.

JoormanN, J. (2006). The relation of rumination and inhibition: Evidence from a negative priming task. Cognitive Therapy \& Research, 30, 149-160.

Joormann, J., DKane, M., \& Gotlib, I. H. (2006). Adaptive and maladaptive components of rumination? Diagnostic specificity and relation to depressive biases. Behavior Therapy, 37, 269-280.

Joormann, J., \& GotLib, I. H. (2008). Updating the contents of working memory in depression: Interference from irrelevant negative material. Journal of Abnormal Psychology, 117, 182-192.

Just, N., \& Alloy, L. B. (1997). The response styles theory of depression: Tests and an extension of the theory. Journal of Abnormal Psychology, 106, 221-229.
Linville, P. (1996). Attention inhibition: Does it underlie ruminative thought? In R. S. Wyer, Jr. (Ed.), Ruminative thoughts: Advances in social cognition (Vol. 9, pp. 121-133). Mahwah, NJ: Erlbaum.

Lyubomirsky, S., \& Nolen-Hoeksema, S. (1993). Self-perpetuating properties of dysphoric rumination. Journal of Personality \& Social Psychology, 65, 339-349.

Minnema, M. T., \& Knowlton, B. J. (2008). Directed forgetting of emotional words. Emotion, 8, 643-652.

Miyake, A., \& Shah, P. (1999). Models of working memory: Mechanisms of active maintenance and executive control. New York: Cambridge University Press.

NeE, D. E., \& Jonides, J. (2008). Dissociable interference-control processes in perception and memory. Psychological Science, 19, 490-500.

NeE, D. E., Jonides, J., \& Berman, M. G. (2007). Neural mechanisms of proactive interference-resolution. NeuroImage, 38, 740-751.

Nolen-Hoeksema, S. (2000). The role of rumination in depressive disorders and mixed anxiety/depressive symptoms. Journal of Abnormal Psychology, 109, 504-511.

Nolen-Hoeksema, S., \& Morrow, J. (1991). A prospective study of depression and posttraumatic stress symptoms after a natural disaster: The 1989 Loma Prieta earthquake. Journal of Personality \& Social Psychology, 61, 115-121.

Nolen-Hoeksema, S., Morrow, J., \& Fredrickson, B. L. (1993). Response styles and the duration of episodes of depressed mood. Journal of Abnormal Psychology, 102, 20-28.

Nolen-Hoeksema, S., Parker, L. E., \& Larson, J. (1994). Ruminative coping with depressed mood following loss. Journal of Personality \& Social Psychology, 67, 92-104.

Nolen-Hoeksema, S., Wisco, B. E., \& Lyubomirsky, S. (2008). Rethinking rumination. Perspectives on Psychological Science, 5, 400-424.

Parrott, W. G., \& Sabini, J. (1990). Mood and memory under natural conditions: Evidence for mood incongruent recall. Journal of Personality \& Social Psychology, 59, 321-336.

Power, M. J., Dalgleish, T., Claudio, V., Tata, P., \& Kentish, J. (2000). The directed forgetting task: Application to emotionally valent material. Journal of Affective Disorders, 57, 147-157.

Purcell, R., Maruff, P., Kyrios, M., \& Pantelis, C. (1997). Neuropsychological function in young patients with unipolar major depression. Psychological Medicine, 27, 1277-1285.

Rose, E. J., \& Ebmeier, K. P. (2006). Pattern of impaired working memory during major depression. Journal of Affective Disorders, 90 , 149-161.

Rusting, C. L., \& DeHaRT, T. (2000). Retrieving positive memories to regulate negative mood: Consequences for mood-congruent memory. Journal of Personality \& Social Psychology, 78, 737-752.

Seibert, P. S., \& Ellis, H. C. (1991). Irrelevant thoughts, emotional mood states, and cognitive task performance. Memory \& Cognition, 19, 507-513.

Siemer, M. (2005). Mood congruent cognitions constitute mood experience. Emotion, 5, 296-308.

Skre, I., Onstad, S., Torgersen, S., \& Kringlen, E. (1991). High interrater reliability for the Structured Clinical Interview for DSM-III-R Axis I (SCID-I). Acta Psychiatrica Scandinavica, 84, 167-173.

Tipper, S. P. (2001). Does negative priming reflect inhibitory mechanisms? A review and integration of conflicting views. Quarterly Journal of Experimental Psychology, 54A, 321-343.

Treynor, W., Gonzalez, R., \& Nolen-Hoeksema, S. (2003). Rumination reconsidered: A psychometric analysis. Cognitive Therapy \& Research, 27, 247-259.

Watkins, E., \& Brown, R. G. (2002). Rumination and executive function in depression: An experimental study. Journal of Neurology, Neurosurgery \& Psychiatry, 72, 400-402.

WENTURA, D. (1999). Activation and inhibition of affective information: Evidence for negative priming in the evaluation task. Cognition \& Emotion, 13, 65-91.

Whitmer, A. J., \& BANICH, M. T. (2007). Inhibition versus switching deficits in different forms of rumination. Psychological Science, 18, 546-553.

Williams, J. B., Gibbon, M., First, M. B., Spitzer, R. L., Davis, M., Borus, J., ET AL. (1992). The Structured Clinical Interview for DSMIII-R (SCID): II. Multisite test-retest reliability. Archives of General Psychiatry, 49, 630-636. 


\section{NOTES}

1. Note that we use the term short-term memory rather than the currently more popular term working memory in our discussions. We do this because the latter term has come to be associated with a model of this memory system that includes currently controversial assumptions (see Jonides et al., 2008, for extensive discussion). Thus, the more neutral term short-term memory seems more appropriate.

2. To examine whether the different rates of comorbid participants in the MDD groups in Experiment 1 and Experiment 2 may have affected our findings, we excluded participants with comorbid diagnoses. This did not change our findings: MDD and CTL groups differed in interference for negative but not positive material in Experiment 1 [negative, $t(40)=3.12, p<.01$; positive, $t(40)<1$, n.s.], but did not differ in interference scores in Experiment 2 [4 letters, $t(28)<1$, n.s.; 8 letters, $t(28)=1.42, p>.05]$.

3. We redid this analysis to ensure that the obtained effects are not due to the group difference in overall response latency (see Faust, Balota, Spieler, \& Ferraro, 1999). We calculated RT proportions by dividing the single RTs by the overall RT for each participant. This transformation did not change our findings: The three-way interaction was still significant $[F(1,45)=8.50, p<.01]$.

(Manuscript received March 11, 2008; revision accepted for publication June 18, 2009.) 